\title{
Polymorphisme de l'alcool déshydrogénase chez le pêcher (Prunus persica L Batsch). Étude génétique
}

\author{
R Monet *, A Guye, M Roy \\ INRA, centre de recherches de Bordeaux, station de recherches fruitières, BP 81, F33883 Villenave-d'Ornon cedex, France
}

(Reçu le 10 juin 1994 ; accepté le 10 octobre 1994)

\begin{abstract}
Résumé - L'alcool déshydrogénase (ADH, EC 1.1.1.1) a été étudié chez le pêcher à partir d'extraits enzymatiques totaux issus de feuilles prélevées en avril et mai (l'activité ADH s'atténue fortement au-delà de cette période). Ce système enzymatique est complexe, il fait intervenir 3 loci : ADH-1, ADH-2 et ADH-3. II se forme à partir de ces 3 loci des enzymes dimères intra- et intergéniques. Le locus $A D H-3$ possède 2 allèles $A D H-3 L$ et $A D H-3 R$ qui ont pu être mis en évidence dans une famille obtenue par l'autofécondation d'un individu hétérozygote.
\end{abstract}

Prunus persica $=$ pêcher $/$ électrophorèse $/$ isozymes $/$ hérédité

Summary - Genetic study of alcohol dehydrogenase polymorphism in peach (Prunus persica L Batsch). Alcohol dehydrogenase (ADH, EC 1.1.1.1) was studied in peach using total protein extracts of leaves. Leaves were harvested from orchard grown trees in April and May, since, after this period, there was a large decrease in ADH activity. The enzymatic system of $A D H$ is complex and involves 3 loci, ie $A D H-1, A D H-2$ and $A D H-3$. From these 3 loci, intraand intergenic dimeric enzymes are formed. The $A D H-3$ locus has 2 alleles, $A D H-3 L$ and $A D H-3 R$. These were both identified within a family resulting from self-pollination of a heterozygous individual.

Prunus persica $=$ peach tree $/$ electrophoresis $/$ isozyme $/$ heredity

\section{INTRODUCTION}

L'alcool déshydrogénase $(A D H)$ est un système enzymatique complexe déterminé le plus souvent par 2 loci structurels comme chez le maïs (Schwartz, 1966), l'orge (Brown, 1980), la tomate (Tanksley, 1979), quelquefois 3 loci comme chez le céleri (Orton, 1983) et le poirier (Nanos et al, 1992).

Chez le blé hexaploïde, Hart (1983) distingue 4 types d'alcool déshydrogénase par leur substrat spécifique et leur cofacteur : l'ADH NAD dépendante qui a pour substrat les alcools aliphatiques primaires et secondaires, l'ADH NADP dépendante qui dégrade les alcools aromatiques, l'ADH sans coenzyme spécifique qui dégrade les alcools aromatiques, enfin l'ADH NAD dépendante qui agit sur les alcools aromatiques. La première de ces enzymes qui correspond à celle que nous étudions sur le pêcher serait contrôlée par 2 loci (Jaaska, 1980).

Certains de ces loci peuvent présenter plusieurs formes alléliques qui vont donner des isozymes différentes (allozymes), le plus souvent 2,

\footnotetext{
* Correspondance et tirés à part.
} 
l'une à migration lente, l'autre à migration rapide. Comme l'enzyme est un dimère, ces allèles vont donner des homo et des hétérodimères ; enfin il peut se former des dimères intergéniques.

Chez le pêcher, 3 études ont pris en compte l'alcool déshydrogénase: Durham et al (1987) n'ont pas décelé d'activité ADH sur des extraits de feuilles d'arbres juvéniles et adultes, Messeguer et al (1987) ont identifié une seule bande à partir d'extraits protéiques de pollen sans polymorphisme intervariétal. Mowrey et Werner (1990) ont identifié 6 bandes sur graines stratifiées pendant 2,5 ou 3 mois et 3 bandes sur feuilles d'arbres adultes. Selon ces auteurs 3 loci pourraient être présents, leur expression serait inductible par des conditions d'anaérobiose.

II faut remarquer que ces 3 auteurs ont pratiqué des électrophorèses sur gel d'amidon dont le pouvoir séparateur est quelquefois insuffisant surtout si le système enzymatique étudié est complexe.

Les essais préliminaires d'électrophorèse en gel d'acrylamide que nous avions réalisés pour étudier l'ADH sur le pêcher avaient révélé un polymorphisme qui permettait de classer les variétés en 2 groupes. L'interprétation de ce polymorphisme n'a seulement été possible que lorsque nous avons pu étudier la descendance par autofécondation d'un individu appartenant à l'un de ces deux groupes et qui était en fait la forme hétérozygote.

Nous présentons donc ici un polymorphisme de l'ADH du pêcher et son mode d'hérédité.

\section{MATÉRIEL ET MÉTHODES}

\section{Le matériel végétal}

Pour illustrer le polymorphisme de l'ADH sur les cultivars de pêcher, nous avons prélevé les échantillons de feuilles destinées à l'électrophorèse sur 44 cultivars assez répandus : Amber Gold, Amsden, Armking, Babygold 5, Babygold 6, Barbara, Dixired, Earlyglo, Everts, Fantasia, Fayette, Flavorcrest, Flavortop, Garnet Beauty, Glohaven, Grand Diamond, Golo ${ }^{\circledR}$, Harko, Henry Moulin, Jalousia ${ }^{\circledR}$, J.H. Hale, Loadel, Loring, Maybelle, Merrill Early O Henry, Merrill Gemfree, Michelini, Niagara, Orion, Peking, Primerose, Redcal, Redhaven, Redjune, Redtop, Redwing, Robin, Royal May, Snowqueen, Southland, Springcrest, Springold, Springtime, Suncrest. Ces clones appartenaient à une collection variétale maintenue sur le domaine expérimental INRA des Jarres. Les arbres étaient âgés de 10 ans et greffés sur le portegreffe Rubira (semis de pêcher).
L'étude génétique s'est faite sur une famille de 240 arbres issus de l'autofécondation du géniteur S2600:56:236, lui même provenant d'un $2^{e}$ cycle d'autofécondation du cultivar Suncrest. Les arbres de cette famille étaient âgés de 5 ans, conduits en parcelle hybride, non greffés, plantés à densité moyenne $(2 \mathrm{x}$ $5 \mathrm{~m}$ ), traités au minimum.

\section{Techniques d'électrophorèse}

Les protéines sont extraites à partir de jeunes feuilles prélevées à la pointe des rameaux en croissance aux mois d'avril et de mai. L'activité ADH s'atténue et disparaît au-delà de cette période. Peu après leur récolte, les jeunes feuilles sont lyophilisées et conservées à $80^{\circ} \mathrm{C}$.

La méthode d'extraction et de purification des protéines a déjà été décrite (Monet et al, 1991). Nous utilisons pour l'électrophorèse des gels à 7,5\% d'acrylamide. Chaque gel, de $1,5 \mathrm{~mm}$ d'épaisseur, $14 \mathrm{~cm}$ de largeur et $13,5 \mathrm{~cm}$ de longueur, permet l'électrophorèse simultanée de 10 échantillons. Le tampon d'électrophorèse a la composition suivante : Tris $\mathrm{HCl} 0,15 \mathrm{~mol}$ $\mathrm{I}^{-1}$, glycine $0,15 \mathrm{~mol} \mathrm{I}^{-1}, \mathrm{pH} 8$. La quantité d'extrait protéique purifié utilisée est de $20 \mu \mathrm{l}$ par puits. L'électrophorèse est verticale, la tension appliquée au gel est de 15 volts par centimètre.

Après migration, l'ADH est révélée par le système Tétrazolium (Vallejos, 1983).

\section{RÉSULTATS}

\section{Polymorphisme de l'ADH chez les cultivars et chez le géniteur S2600:56:236}

Les 44 cultivars étudiés se répartissent en 2 groupes:

- un groupe majoritaire ayant un phénotype à 6 bandes (fig 1A) qui comprend les variétés suivantes : Amber Gold, Amsden, Armking, Babygold 5, Babygold 6, Barbara, Earlyglo, Everts, Fantasia, Fayette, Flavorcrest, Flavortop, Garnet Beauty, Grand Diamond, Golo ${ }^{\circledR}$, Henry Moulin, Jalousia ${ }^{\circledR}$, JH Hale, Loadel, Loring, Maybelle, Merrill Early O Henry, Merrill Gemfree, Michelini, Niagara, Orion, Peking, Primerose, Redcal, Redjune, Redtop, Redwing, Robin, Royal May, Snowqueen, Southland, Springcrest, Springold, Springtime;

- un groupe minoritaire ayant un phénotype à 9 bandes (fig 1B) qui comprend les variétés suivantes: Dixired, Glohaven, Harko, Redhaven, Suncrest.

Le géniteur S2600:56:236, issu d'un second cycle d'autofécondation de Suncrest, présente, comme ce dernier, 9 bandes (fig 1B). 


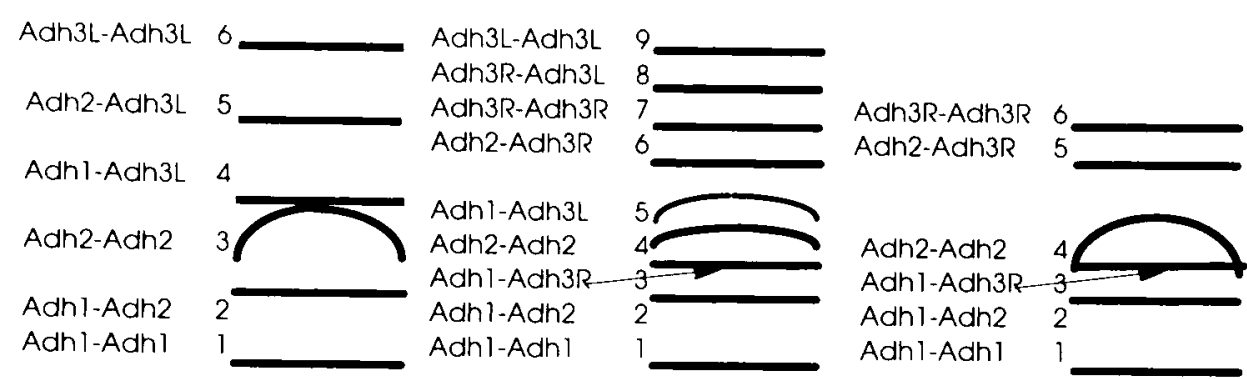

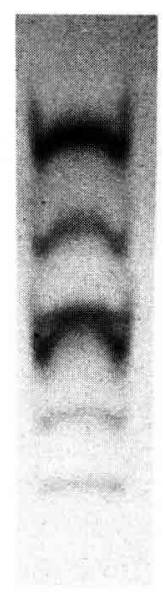

A

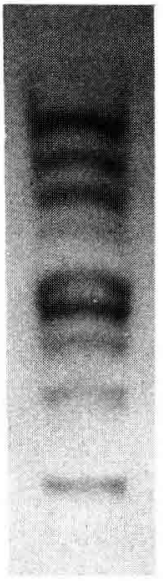

B

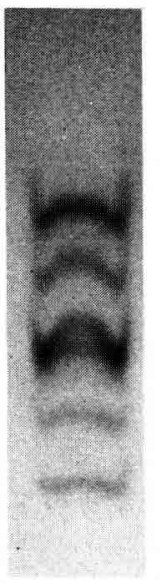

C

Fig 1. Les 3 profils du système enzymatique $A D H$ chez le pêcher et leur interprétation schématique. Le profil $A$ est celui de l'ADH-3 à migration lente, le profil $\mathrm{C}$ est celui de l'ADH-3 à migration rapide, le profil $\mathrm{B}$ correspond à l'état hétérozygote.

\section{Etude de la famille issue de l'autofécondation de S2600:56:236}

Les individus de cette famille se répartissent en 3 catégories: ceux qui ont 9 bandes comme le parent, ceux qui ont 6 bandes comme on les trouve chez les cultivars étudiés ci-dessus, enfin une nouvelle catégorie d'individus à 6 bandes (fig 1C) mais dont l'espacement est différent du précédent. En effet, si on numérote de 1 à 6 les bandes, de la plus rapide à la plus lente, pour chaque individu la bande 1 se trouve toujours au même niveau du zymogramme ; en revanche la bande 6 est plus éloignée de l'origine de la migration chez la nouvelle catégorie d'individus ; il en résulte un resserrement différent des bandes intermédiaires 2 et 3 . Si nous observons que l'une de ces bandes intermédiaires est particulièrement incurvée (bande 4 du profil A), nous voyons que la bande qui était en position 3 dans ce même profil est passée en position 4 dans le profil $C$. Nous pouvons donc distinguer, dans la famille, les individus à 6 bandes dont la bande 6 est à migration lente (fig 1A), les individus à 6 bandes, dont la bande
6 est à migration rapide (fig $1 \mathrm{C}$ ) et les individus à 9 bandes (fig 1B).

Les proportions des 3 catégories d'arbres sont assimilables à une disjonction mendélienne de type 1:2:1 (voir tableau I).

On peut donc dire que le géniteur à 9 bandes, S2600:56:236, est hétérozygote et a pour ancêtres (qu'il faudrait rechercher en amont de Suncrest) un parent à 6 bandes de profil $A$ (bande 6 lente) et un parent à 6 bandes de profil $\mathrm{B}$ (bande 6 rapide).

Tableau I. Répartition des individus selon les 3 phénotypes de I'ADH dans une famille issue de l'autofécondation du géniteur S2600:56:236.

$\begin{array}{cccc}\text { Phénotypes } & \begin{array}{l}6 \text { bandes } \\ \text { (bande } \\ 6 \text { lente) }\end{array} & 9 \text { bandes } & \begin{array}{l}6 \text { bandes } \\ \text { (bande } \\ 6 \text { rapide) } \\ -\end{array} \\ \text { Fréquences } & 68(60) & 120(120) & 52(60)\end{array}$

Les chiffres entre parenthèses sont les valeurs théoriques pour une disjonction 1:2:1 $\left(\chi^{2} \mathrm{ob}=2,14 ; \chi^{2}\right.$ th $=5,99$ pour 2 degrés de liberté, non significatif). 


\section{DISCUSSION}

L'ADH étant un dimère, l'interprétation des profils observés nécessite l'intervention de 3 loci fonctionnels ADH-1, ADH-2 et ADH-3 (le locus donnant la bande la plus rapide est numéroté 1) et 2 allèles sur le locus ADH-3: $A D H-3 L$ (lent) et ADH-3R (rapide) puisque nous avons obtenu dans la famille S2600:56:236 une disjonction typiquement mendélienne de type 1:2:1. Les différentes bandes observées résultent de la formation de dimères d'origine intra- ou intergénique.

En donnant le même symbole à l'allèle et à la chaîne polypeptidique qu'il génère, l'interprétation des zymogrammes est donnée dans les schémas de la figure 1 . Nous avons des bandes qui correspondent à des homodimères intragéniques ou intra-alléliques exemples : $A D H-1 / A D H-1, A D H$ $3 \mathrm{~L} / \mathrm{ADH}-3 \mathrm{~L}$ et des bandes qui correspondent à des hétérodimères inter-géniques ou inter-alléliques exemples : $A D H-1 / A D H-2, A D H-3 R / A D H-$ 3L. II manque, dans le profil $B$ relatif aux génotypes hétérozygotes, l'hétérodimère intergénique $\mathrm{ADH}-2 / \mathrm{ADH}-3 \mathrm{R}$ : cet hétérodimère est confondu avec l'homodimère ADH-3/ADH-3.

Nous n'avons pas, dans cette famille qui est en $3^{e}$ cycle d'autofécondation, d'autres caractères mendéliens en disjonction qui auraient permis d'étudier leur liaison génétique avec le locus de l'ADH3.

\section{CONCLUSION}

L'alcool déshydrogénase est, chez le pêcher, un système enzymatique complexe qui nécessite, pour son interprétation, des zymogrammes de bonne qualité, ce qui explique peut-être que son polymorphisme soit passé inaperçu jusqu'ici. $\mathrm{Ce}$ système est par ailleurs inductible ; s'il est présent dans les extraits de jeunes feuilles, il s'atténue très vite quand les feuilles vieillissent. L'intensité des zymogrammes peut aussi varier d'un arbre à l'autre, cela peut rendre leur lecture difficile si l'on n'a pas déjà identifié les 3 systèmes existants. Enfin, ce polymorphisme est mal réparti ; la majorité des variétés que nous avons étudiées possède le profil $A$; quelquesunes sont hétérozygotes mais 3 d'entre elles (Dixired, Glohaven, Redhaven) possèdent un ancêtre commun; nous n'avons pas trouvé de variétés à profil $C$ dans l'échantillon aléatoire de 44 cultivars, que nous avons étudié.

L'interprétation génétique la plus vraisemblable des zymogrammes, compte tenu des 3 profils identifiés et de la disjonction que présente la famille issue de l'autofécondation du géniteur S2600:56:236, fait intervenir 3 loci : ADH1, ADH2 et $\mathrm{ADH} 3$ ce dernier possédant 2 allèles.

On considère le pêcher comme étant une espèce peu polymorphe. L'autogamie préférentielle de celle-ci a vraisemblablement contribué à créer des isolats entre lesquels les échanges génétiques sont limités. Les variétés cultivées ont été obtenues en exploitant de préférence certains de ces isolats, ce qui explique leurs bases génétiques étroites et donc leur faible polymorphisme. Ainsi l'exploration plus complète de l'espèce pourrait révéler plus de diversité que prévu. La découverte du polymorphisme de l'ADH est encourageante de ce point de vue.

\section{RÉFÉRENCES}

Brown AHD (1980) Genetic basis of alcohol dehydrogenase polymorphism in Hordeum spontaneum. $J$ Heredity 70, 127-128

Durham RE, Moore GA, Sherman WB (1987) Isozyme banding patterns and their usefulness as genetic markers in peach. J Amer Soc Hort Sci 112, 1013-1018

Hart GE (1983) Hexaploïd wheat (Triticum aestivum $\mathbf{L}$ em Thell). In : Isozymes in plant genetics and breeding part $B$ (SD Tanksley, TJ Orton, eds), Elsevier, Amsterdam, 35-56

Jaaska V (1980) Anaerobic induction of alcohol dehydrogenase isoenzyme in tetraploid wheats and their diploid relatives. Biochem Physiol Pflanzen 175, 570-577

Messeguer R, Arus P, Carrera M (1987) Identification of peach cultivars with pollen isozymes. Sci Hortic 31, 107-117

Monet R, Gibault B (1991) Polymorphisme de l' $\alpha$-amylase chez le pêcher. Étude génétique. agronomie 11, 353-358

Mowrey BD, Werner DJ (1990) Developmental specific isozyme expression in peach. Hortscience 25,219 222

Nanos GD, Romani RJ, Kader AA (1992) Metabolic and other responses of "Bartlett" pear fruit and suspension cultured "Passe Crassane" pear fruit cells held in $0.25 \% \mathrm{O}_{2}$. J Amer Soc Hort Sci 117, 934-940

Orton TJ (1983) Celery and celeriac (Apium graveolens $\mathrm{L}$ ). In : Isozymes in plant genetics and breeding part B (SD Tanksley, TJ Orton, eds), Elsevier, Amsterdam, 351-367

Schwartz D (1966) The genetic control of alcohol dehydrogenase in maize gene duplication and regression. Proc Natl Acad Sci USA 56, 1431-1436

Tanksley SD (1979) Linkage, chromosomal association and expression of ADH-1 and PGM-2 in tomato. Biochem Genet 17, 1159-1167

Vallejos CE (1983) Enzyme activity staining. In : Isozymes in plant genetics and breeding part A (SD Tanksley, TJ Orton, eds), Elsevier, Amsterdam, 469-516 